\title{
Torsional Oscillations Excited by Feeding in Subsynchronous Currents from the Grid into the Electrical Generator
}

\author{
Matthias Humer \\ Produktgruppe Messtechnik/Product Group Metrology Uniper, Germany \\ Email: matthias.humer@uniper.energy
}

How to cite this paper: Humer, M. (2020) Torsional Oscillations Excited by Feeding in Subsynchronous Currents from the Grid into the Electrical Generator. Open Access Library Journal, 7: e7035.

https://doi.org/10.4236/oalib.1107035

Received: November 25, 2020

Accepted: December 19, 2020

Published: December 22, 2020

Copyright $\odot 2020$ by author(s) and Open Access Library Inc.

This work is licensed under the Creative Commons Attribution International License (CC BY 4.0).

http://creativecommons.org/licenses/by/4.0/

\section{(c) (i) Open Access}

\begin{abstract}
In the following article, results of the investigation on the effect of feeding subsynchronous currents from the electric grid in the generator are presented. In the end, torsional oscillations of the shaft train are excited, which can reach very high amplitudes in case of resonance. In time with the torsional oscillations, the synchronous generated voltage induced in the stator winding oscillates, which leads to the feed of subsynchronous currents from the generator into the electrical network. The focus is directed to the effect of these currents in the system "generator-grid". Therefore, different analytical approaches to subsynchronous power excitation are presented and evaluated. In particular, the analytical equations of generator power generated by subsynchronous electric currents are derived. This article shows that, in addition to the damping properties in dealing with torsional oscillations, the interaction between the grid frequency, the natural electrical grid frequencies and the mechanical torsional natural frequencies is also important.
\end{abstract}

\section{Subject Areas}

Electric Engineering

\section{Keywords}

Torsion, Electrical Grid, Electrical Generator, UIF, Subsynchronous

Excitation, Converter

\section{Introduction}

Due to the steady increase, see Figure 1, of converters and converter systems in the German transmission grid, e.g. due to the steady expansion of offshore wind 


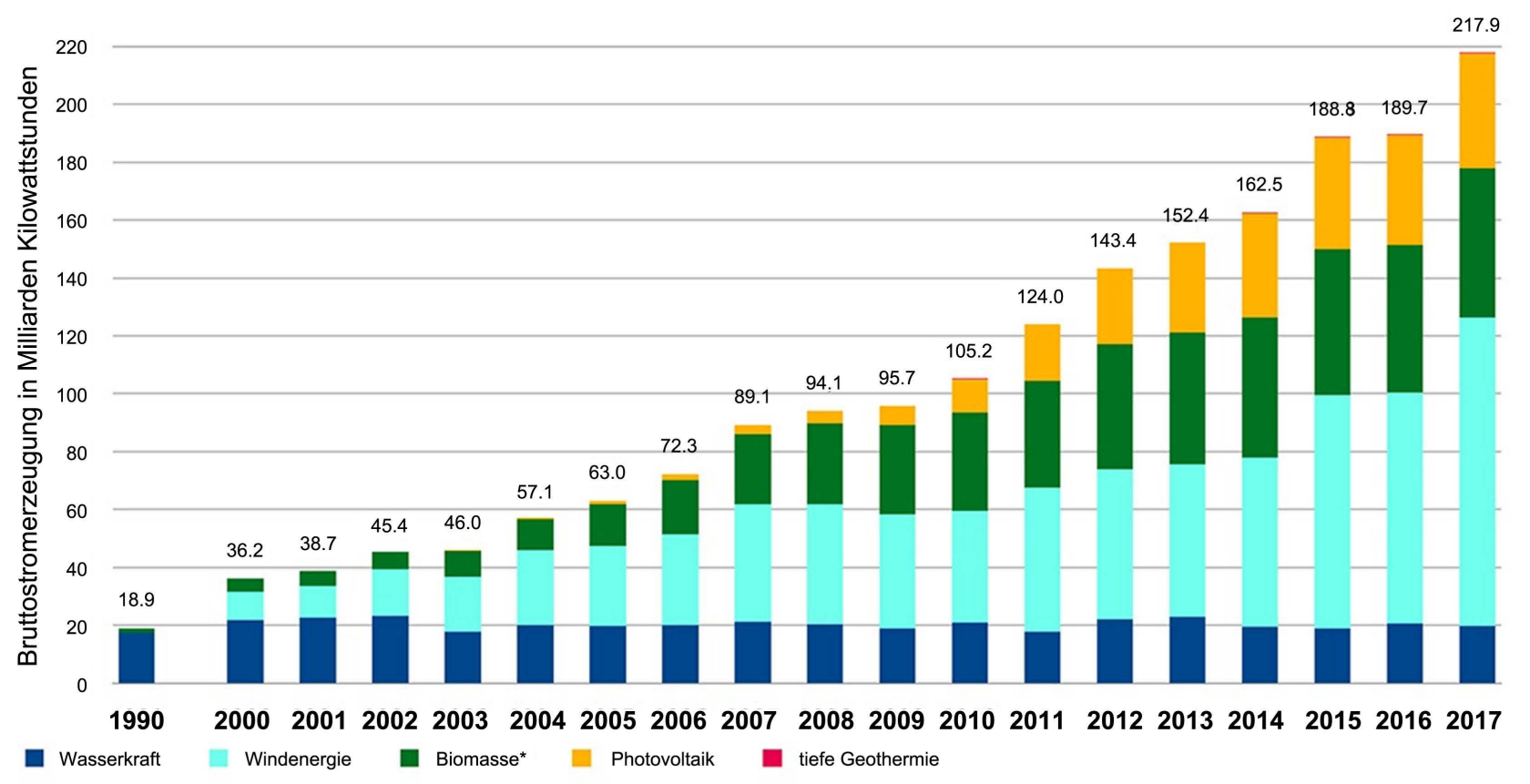

* inkl. feste und flussige Biomasse, Biogas inkl. Biomethan, Klar- und Deponiegas und dem biogenen Anteil des Abfalls, ab 2010 inkl. Klarschlamm; BMWi auf Basis Arbeitsgruppe Erneuerbare Energien Statistik (AGEE Stat); Stand: Februar 2018; Angaben vorlaufig

Figure 1. Increasing number of frequency converters in Germany (Source: BMWI).

farms and solar power, it is necessary to deal with the grid based excitation of torsional oscillations of the turbo sets by sub-synchronously fed-in currents. It is to be expected that there will be an increase of sub-synchronous feeding processes through frequency converters into the transmission grid in the future. These processes will then lead to subsynchronous power oscillations between the grid and the generator, which in turn may excite subsynchronous torsional natural frequencies of the turboset shaft trains. This problem is currently being investigated and discussed in Germany, e.g. within an expert committee under the leadership of the VGB PowerTech e.V. A decisive question here is the ability of frequency converters to excite or to damp torsional oscillations. For this purpose, extensive simulations, which include both grid models and turboset models, are carried out by the grid operators. The central criterion for deciding whether the turboset of a power plant could be affected by the connection of a frequency converter is the value of the "Unit Interaction Factor" (UIF).

If the value of the UIF is below 0.01 , there is no risk of negative effects on the turbo set by switching on a frequency converter. The UIF itself is calculated according to [1] [2] as follows, see Equation (1):

$$
\mathrm{UIF}=\frac{M W_{d c}}{M V A_{g}} \cdot\left(1-\frac{S C_{g}}{S C_{\text {tot }}}\right)^{2}
$$

Herein mean:

$M W_{d c}$ : DC nominal power of the frequency converter,

$M V A_{g}$ : Nominal apparent power of the generator, 
$S C_{\text {tot }}$ : Short-circuit capacity at the DC commutation node including the generator,

$S C_{g}$ : Short-circuit capacity at the DC commutation node without the generator.

\section{Effect of the Feed in of Sub-Frequency Currents on the Generator Power}

The starting point for the following considerations is the definitions of the currents and voltages of the SSR feed-in model according to Figure 2. Based on this model, the generator power at the terminals is first derived in a single-phase state and then extended to the three-phase state.

The currents and voltages shown in Figure 2 have the following meaning:

Single-phase stator voltage with the grid frequency $f_{N}$ :

$$
u(t)=\hat{u} \cdot \sin \left(\omega_{N} \cdot t+\varphi_{u}\right) \text { mit } \omega_{N}=2 \cdot \pi \cdot f_{N}
$$

Single-phase stator current with the grid frequency $f_{N}$ :

$$
i(t)=\hat{i} \cdot \sin \left(\omega_{N} \cdot t+\varphi_{i}\right) \text { mit } \omega_{N}=2 \cdot \pi \cdot f_{N}
$$

Sub-frequency current with the frequency $f_{S S R 1}$, which is fed from the grid and flows into the generator:

$$
\begin{gathered}
i(t)_{S S R 1}=\hat{i}_{S S R 1} \cdot \mathrm{e}^{-\alpha_{S S R 1} \cdot t} \cdot \sin \left(\omega_{S S R 1} \cdot t+\varphi_{S S R 1}\right) \\
\text { with } \omega_{S S R 1}=2 \cdot \pi \cdot f_{S S R 1} \text { and } \alpha_{S S R 1}=\text { damping factor }
\end{gathered}
$$

Due to the excited torsional oscillations, angular oscillations of the generator rotor occur with a natural torsional frequency $f_{S S R 2}$. This additionally induces a voltage in the stator winding, which produces a sub-frequency current with the frequency $f_{S S R 2}$ :

$$
\begin{gathered}
i(t)_{S S R 2}=\hat{i}_{S S R 2} \cdot \mathrm{e}^{-\alpha_{S S R 2} \cdot t} \cdot \sin \left(\omega_{S S R 2} \cdot t+\varphi_{S S R 2}\right) \\
\text { with } \omega_{S S R 2}=2 \cdot \pi \cdot f_{S S R 2} \text { and } \alpha_{S S R 2}=\text { damping factor }
\end{gathered}
$$

The used index "1" (cause) or "2" (effect) shall only allow a differentiation

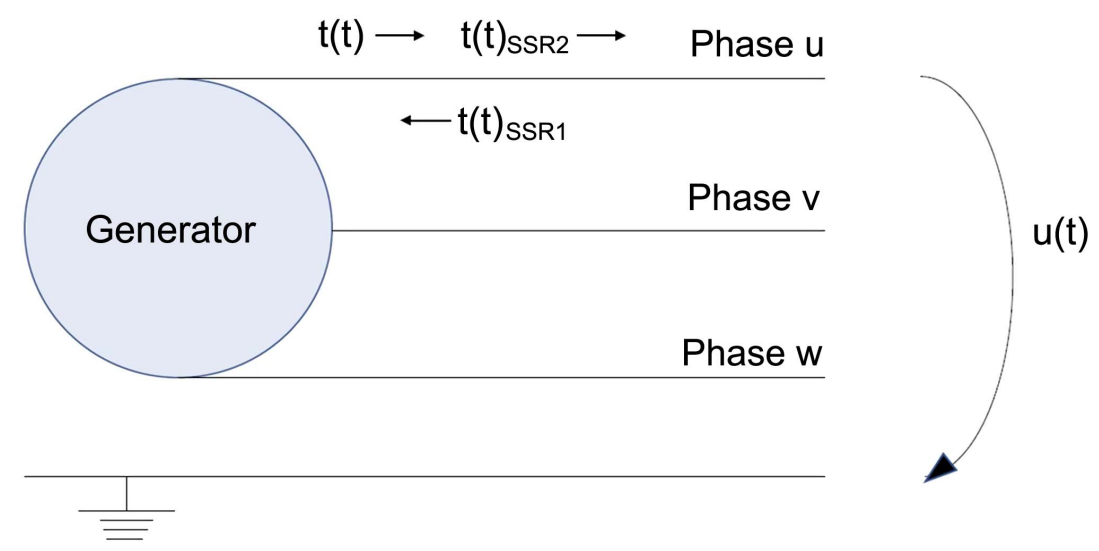

Figure 2. Basis of the SSR feed-in model. 
between these two states at this point. Furthermore it was assumed that the current $i_{S S R 2 \text { rms }}$ opposite to the current $i_{S S R 1 r m s}$ flows. This state occurs if the condition $\Delta \varphi_{S S R 2}=\varphi_{S S R 1}+180^{\circ}$ is fulfilled, where $\Delta \varphi_{S S R 2}$ a part of the phase angle $\varphi_{S S R 2}$ represents. Starting from Figure 2 the single-phase power $p(t)$ is now formed at a generator terminal e.g. of phase $u$ :

$$
\begin{aligned}
p_{u}(t) & =u(t) \cdot\left[i(t)+i(t)_{S S R 1}+i(t)_{S S R 2}\right] \\
& =\underbrace{u(t) \cdot i(t)}_{p_{u 1}(t)}+\underbrace{u(t) \cdot i(t)_{S S R 1}}_{p_{u 2}(t)}+\underbrace{u(t) \cdot i(t)_{S S R 2}}_{p_{u 3}(t)}
\end{aligned}
$$

In the following, each summand from Equation (2) is calculated individually to ensure clarity.

$$
\begin{aligned}
p_{u 1}(t) & =u(t) \cdot i(t)=\frac{1}{2} \cdot \hat{u} \cdot \hat{i} \cdot\left[\cos \left(\varphi_{u}-\varphi_{i}\right)-\cos \left(2 \cdot \omega_{N} \cdot t+\varphi_{u}+\varphi_{i}\right)\right] \\
& =u_{r m s} \cdot i_{r m s} \cdot \cos (\Delta \varphi)-u_{r m s} \cdot i_{r m s} \cdot \cos \left(2 \cdot \omega_{N} \cdot t+\varphi_{u}+\varphi_{i}\right)
\end{aligned}
$$

Equation (3) describes the mean value of the instantaneous power as a function of $\cos (\Delta \varphi)$ and the double line frequency power oscillation.

$$
\begin{aligned}
p_{u 2}(t)= & u(t) \cdot i(t)_{S S R 1} \\
= & \hat{u} \cdot \hat{i}_{S S R 1} \cdot \mathrm{e}^{-\alpha_{S S R 1} \cdot t} \cdot \sin \left(\omega_{N} \cdot t+\varphi_{u}\right) \cdot \sin \left(\omega_{S S R 1} \cdot t+\varphi_{S S R 1}\right) \\
= & \frac{1}{2} \hat{u} \cdot \hat{i}_{S S R 1} \cdot \mathrm{e}^{-\alpha_{S S R 1} \cdot t} \cdot\left[\cos \left(\omega_{N} \cdot t+\varphi_{u}-\omega_{S S R 1} \cdot t-\varphi_{S S R 1}\right)\right. \\
& \left.-\cos \left(\omega_{N} \cdot t+\varphi_{u}+\omega_{S S R 1} \cdot t+\varphi_{S S R 1}\right)\right] \\
= & u_{r m s} \cdot i_{S S R 1 r m s} \cdot \mathrm{e}^{-\alpha_{S S R 1} \cdot t} \cdot\left[\cos \left(\left(\omega_{N}-\omega_{S S R 1}\right) \cdot t+\left(\varphi_{u}-\varphi_{S S R 1}\right)\right)\right. \\
& \left.-\cos \left(\left(\omega_{N}+\omega_{S S R 1}\right) \cdot t+\left(\varphi_{u}+\varphi_{S S R 1}\right)\right)\right]
\end{aligned}
$$

Equation (4) contains the fluctuating power component with the frequency $f_{S S R 1}$, which is fed from the grid and excites the generator rotor to torsional oscillations.

$$
\begin{aligned}
p_{u 3}(t)= & u(t) \cdot i(t)_{S S R 2} \\
= & \hat{u} \cdot \hat{i}_{S S R 2} \cdot \mathrm{e}^{-\alpha_{S S R 2} \cdot t} \cdot \sin \left(\omega_{N} \cdot t+\varphi_{u}\right) \cdot \sin \left(\omega_{S S R 2} \cdot t+\varphi_{S S R 2}\right) \\
= & \frac{1}{2} \hat{u} \cdot \hat{i}_{S S R 2} \cdot \mathrm{e}^{-\alpha_{S S R 2} \cdot t} \cdot\left[\cos \left(\omega_{N} \cdot t+\varphi_{u}-\omega_{S S R 2} \cdot t-\varphi_{S S R 2}\right)\right. \\
& \left.-\cos \left(\omega_{N} \cdot t+\varphi_{u}+\omega_{S S R 2} \cdot t+\varphi_{S S R 2}\right)\right] \\
= & u_{r m s} \cdot i_{S S R 2 r m s} \cdot \mathrm{e}^{-\alpha_{S S R 2} \cdot t} \cdot\left[\cos \left(\left(\omega_{N}-\omega_{S S R 2}\right) \cdot t+\left(\varphi_{u}-\varphi_{S S R 2}\right)\right)\right. \\
& \left.-\cos \left(\left(\omega_{N}+\omega_{S S R 2}\right) \cdot t+\left(\varphi_{u}+\varphi_{S S R 2}\right)\right)\right]
\end{aligned}
$$

Equation (5) represents the fluctuating power component, which is generated by the excited torsional oscillations of the generator rotor and fed into the grid. This process takes place with the frequency $f_{S S R 2}$. The addition of the terms from Equations (3)-(5) leads to the sum in Equation (6):

$$
\begin{aligned}
p_{u}(t) & =u(t) \cdot\left[i(t)+i(t)_{S S R 1}+i(t)_{S S R 2}\right] \\
& =\underbrace{u(t) \cdot i(t)}_{p_{u 1}(t)}+\underbrace{u(t) \cdot i(t)_{S S R 1}}_{p_{u 2}(t)}+\underbrace{u(t) \cdot i(t)_{S S R 2}}_{p_{u 3}(t)}
\end{aligned}
$$




$$
\begin{aligned}
= & u_{r m s} \cdot i_{r m s} \cdot \cos (\Delta \varphi)-u_{e f f} \cdot i_{e f f} \cdot \cos \left(2 \cdot \omega_{N} \cdot t+\varphi_{u}+\varphi_{i}\right) \\
& +u_{r m s} \cdot i_{S S R 1 r m s} \cdot \mathrm{e}^{-\alpha_{S S R 1} \cdot t} \cdot\left[\cos \left(\left(\omega_{N}-\omega_{S S R 1}\right) \cdot t+\left(\varphi_{u}-\varphi_{S S R 1}\right)\right)\right. \\
& \left.-\cos \left(\left(\omega_{N}+\omega_{S S R 1}\right) \cdot t+\left(\varphi_{u}+\varphi_{S S R 1}\right)\right)\right] \\
& +u_{r m s} \cdot i_{S S R 2 r m s} \cdot \mathrm{e}^{-\alpha_{S S R 2} \cdot t} \cdot\left[\cos \left(\left(\omega_{N}-\omega_{S S R 2}\right) \cdot t+\left(\varphi_{u}-\varphi_{S S R 2}\right)\right)\right. \\
& \left.-\cos \left(\left(\omega_{N}+\omega_{S S R 2}\right) \cdot t+\left(\varphi_{u}+\varphi_{S S R 2}\right)\right)\right]
\end{aligned}
$$

By feeding in subsynchronous currents, components with new frequencies in the power components are created in the single-phase total power, see Equation (6), which lie below and above the line frequency, namely $\omega_{N}-\omega_{S S R 1}, \omega_{N}+\omega_{S S R 1}$, $\omega_{N}-\omega_{S S R 2}, \omega_{N}+\omega_{S S R 2}$. In the following, only terms with subsynchronous frequencies will be considered more closely. The excitation of a torsion natural frequency $f_{S S R 2}$ of the shaft train takes place exactly when the following condition is fulfilled:

- $\omega_{N}-\omega_{S S R 1}=\omega_{S S R 2}$ resp. $f_{N}-f_{S S R 1}=f_{S S R 2}$

$\bigcirc \quad f_{S S R 2}$ : excited subsynchronous torsional natural frequency of the shaft train. (7)

This is a necessary condition. In addition, the following optional condition of the system consisting of the turboset and the electrical grid shall apply:

- $\omega_{N}-\omega_{S S R 2}=\omega_{N e f}$ resp. $f_{N}-f_{S S R 2}=f_{N e f}$

$\bigcirc f_{N e f}$ : existing natural grid frequency of the electrical grid.

The combination of Equation (7) with Equation (7a) then results in Equation (7b):

$$
\Rightarrow \omega_{N}-\omega_{S S R 2}=\omega_{N e f} \Leftrightarrow \omega_{N}-\omega_{N}+\omega_{S S R 1}=\omega_{N e f} \Leftrightarrow \omega_{S S R 1}=\omega_{N e f}
$$

From Equation ( $7 \mathrm{~b}$ ) it can be deduced that the currents fed into the generator from the grid occur with a sub-frequency grid natural frequency.

\subsection{Extension to a Three-Phase System}

The next step is the extension of the single-phase to the three-phase view under respect of the $120^{\circ}$ electrical phse shift. The starting point for this is Equation (6). Under the assumption that the current and voltage amplitudes are the same in each case, which differs only by the corresponding phase angles, Equation (8) is obtained:

$$
\begin{aligned}
p(t)= & u(t) \cdot\left[i(t)+i(t)_{S S R 1}+i(t)_{S S R 2}\right]_{u, v, w} \\
= & \underbrace{u(t) \cdot i(t))_{u, v, w}}_{p_{1}(t)_{u, v, w}}+\underbrace{\left(u(t) \cdot i(t)_{S S R)_{u, v, w}}\right.}_{p_{2}(t)_{u, v, w}}+\underbrace{\left(u(t) \cdot i(t)_{S S R 2}\right)_{u, v, w}}_{p_{3}(t)_{u, v, w}} \\
= & u_{r m s} \cdot i_{r m s} \cdot \cos (\Delta \varphi)-u_{r m s} \cdot i_{r m s} \cdot \cos \left(2 \cdot \omega_{N} \cdot t+\varphi_{i}\right) \\
& +u_{r m s} \cdot i_{r m s} \cdot \cos (\Delta \varphi)-u_{r m s} \cdot i_{r m s} \cdot \cos \left(2 \cdot \omega_{N} \cdot t+120^{\circ}+\varphi_{i}+120^{\circ}\right) \\
& +u_{r m s} \cdot i_{r m s} \cdot \cos (\Delta \varphi)-u_{r m s} \cdot i_{r m s} \cdot \cos \left(2 \cdot \omega_{N} \cdot t+240^{\circ}+\varphi_{i}+240^{\circ}\right) \\
& +u_{r m s} \cdot i_{S S R 1 r m s} \cdot \mathrm{e}^{-\alpha_{S S R 1} \cdot t} \cdot\left[\cos \left(\left(\omega_{N}-\omega_{S S R 1}\right) \cdot t+\left(-\varphi_{S S R 1}\right)\right)\right. \\
& \left.-\cos \left(\left(\omega_{N}+\omega_{S S R 1}\right) \cdot t+\left(+\varphi_{S S R 1}\right)\right)\right]
\end{aligned}
$$




$$
\begin{aligned}
& +u_{r m s} \cdot i_{S S R 1 r m s} \cdot \mathrm{e}^{-\alpha_{S S R 1} \cdot t} \cdot\left[\cos \left(\left(\omega_{N}-\omega_{S S R 1}\right) \cdot t+\left(120^{\circ}-120^{\circ}-\varphi_{S S R 1}\right)\right)\right. \\
& \left.-\cos \left(\left(\omega_{N}+\omega_{S S R 1}\right) \cdot t+\left(120^{\circ}+120^{\circ}+\varphi_{S S R 1}\right)\right)\right] \\
& +u_{r m s} \cdot i_{S S R 1 r m s} \cdot \mathrm{e}^{-\alpha_{S S R 1} \cdot t} \cdot\left[\cos \left(\left(\omega_{N}-\omega_{S S R 1}\right) \cdot t+\left(240^{\circ}-240^{\circ}-\varphi_{S S R 1}\right)\right)\right. \\
& \left.-\cos \left(\left(\omega_{N}+\omega_{S S R 1}\right) \cdot t+\left(240^{\circ}+240^{\circ}+\varphi_{S S R 1}\right)\right)\right] \\
& +u_{r m s} \cdot i_{S S R 2 r m s} \cdot \mathrm{e}^{-\alpha_{S S R 2} \cdot t} \cdot\left[\cos \left(\left(\omega_{N}-\omega_{S S R 2}\right) \cdot t+\left(-\varphi_{S S R 2}\right)\right)\right. \\
& \left.-\cos \left(\left(\omega_{N}+\omega_{S S R 2}\right) \cdot t+\left(+\varphi_{S S R 2}\right)\right)\right] \\
& +u_{r m s} \cdot i_{S S R 2 r m s} \cdot \mathrm{e}^{-\alpha_{S S R 2} \cdot t} \cdot\left[\cos \left(\left(\omega_{N}-\omega_{S S R 2}\right) \cdot t+\left(120^{\circ}-120^{\circ}-\varphi_{S S R 2}\right)\right)\right. \\
& \left.-\cos \left(\left(\omega_{N}+\omega_{S S R 2}\right) \cdot t+\left(120^{\circ}+120^{\circ}+\varphi_{S S R 2}\right)\right)\right] \\
& +u_{r m s} \cdot i_{S S R 2 r m s} \cdot \mathrm{e}^{-\alpha_{S S R 2} \cdot t} \cdot\left[\cos \left(\left(\omega_{N}-\omega_{S S R 2}\right) \cdot t+\left(240^{\circ}-240^{\circ}-\varphi_{S S R 2}\right)\right)\right. \\
& \left.-\cos \left(\left(\omega_{N}+\omega_{S S R 2}\right) \cdot t+\left(240^{\circ}+240^{\circ}+\varphi_{S S R 2}\right)\right)\right]
\end{aligned}
$$

After transforming and reducing the equation, the final result is:

$$
\begin{aligned}
p(t)= & 3 \cdot u_{r m s} \cdot i_{r m s} \cdot \cos (\Delta \varphi) \\
& +3 \cdot u_{r m s} \cdot i_{S S R 1 r m s} \cdot \mathrm{e}^{-\alpha_{S S R 1} \cdot t} \cdot\left[\cos \left(\left(\omega_{N}-\omega_{S S R 1}\right) \cdot t-\varphi_{S S R 1}\right)\right] \\
& +3 \cdot u_{r m s} \cdot i_{S S R 2 r m s} \cdot \mathrm{e}^{-\alpha_{S S R 2} \cdot t} \cdot\left[\cos \left(\left(\omega_{N}-\omega_{S S R 2}\right) \cdot t-\varphi_{S S R 2}\right)\right]
\end{aligned}
$$

The first summand in Equation (8) stands for the stationary power component. The second summand describes the subsynchronous power oscillation, which is generated in the generator by the sub-frequency currents fed from the grid. The third summand contains the subsynchronous power oscillation, which is emitted from the generator into the grid. The damping factor $\alpha$ is of decisive importance here, since it determines whether the power oscillation is stationary with sub-synchronous power oscillations or whether the sub-synchronous power oscillations decay over time.

\subsection{Variation in Time of the Three-Phase Power}

To illustrate the effects of the power oscillations caused by the sub-frequency currents fed into the system, a corresponding scenario is shown in Figure 3 and Figure 4. Figure 3 shows the power oscillation for 500 time cycles, Figure 4 shows the same scenario for a section of 100 time cycles. Here the process of frequency modulation is clearly visible. The power oscillates with an active power of 0.7 p.u. The sub-synchronous active power fed into the generator from the $50 \mathrm{~Hz}$ grid is 0.05 p.u. with a frequency of $33 \mathrm{~Hz}$ and a damping factor of 0.003 . The sub-synchronous active power fed into the $50 \mathrm{~Hz}$ grid from the generator is 0.04 p.u. with a frequency of $17 \mathrm{~Hz}$ and a damping factor of 0.002 . Furthermore, a frequency of 1.0 p.u. was used, with 0.7 p.u., with 0.05 p.u. and with 0.04 p.u.

\subsection{Selected Boundary Conditions for the Excitation}

Taking Equation (8) into account, various excitation cases for 3-phase operation using the frequency ratios are presented below. 


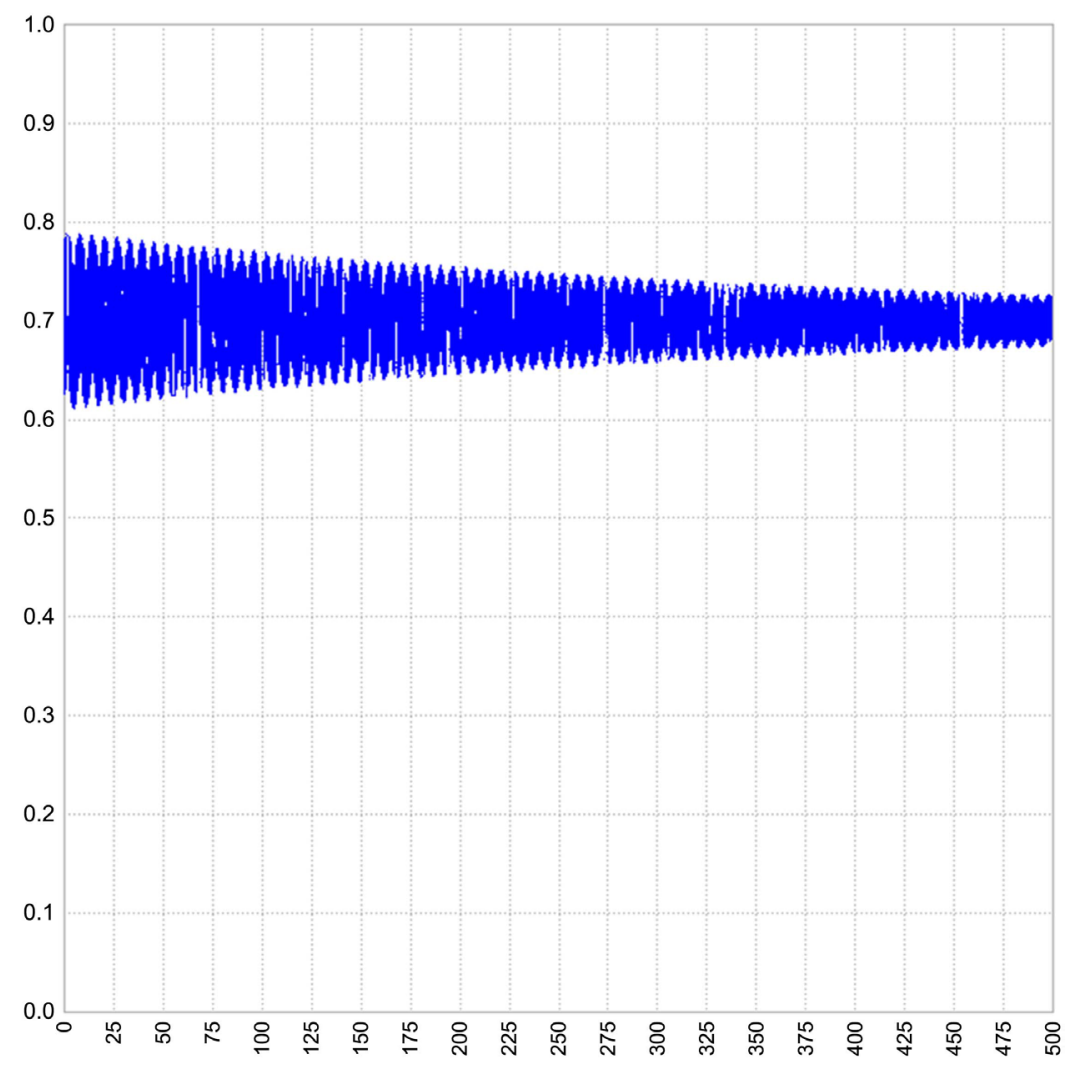

Figure 3. SSR power oscillation via 500 time cycles in p.u.

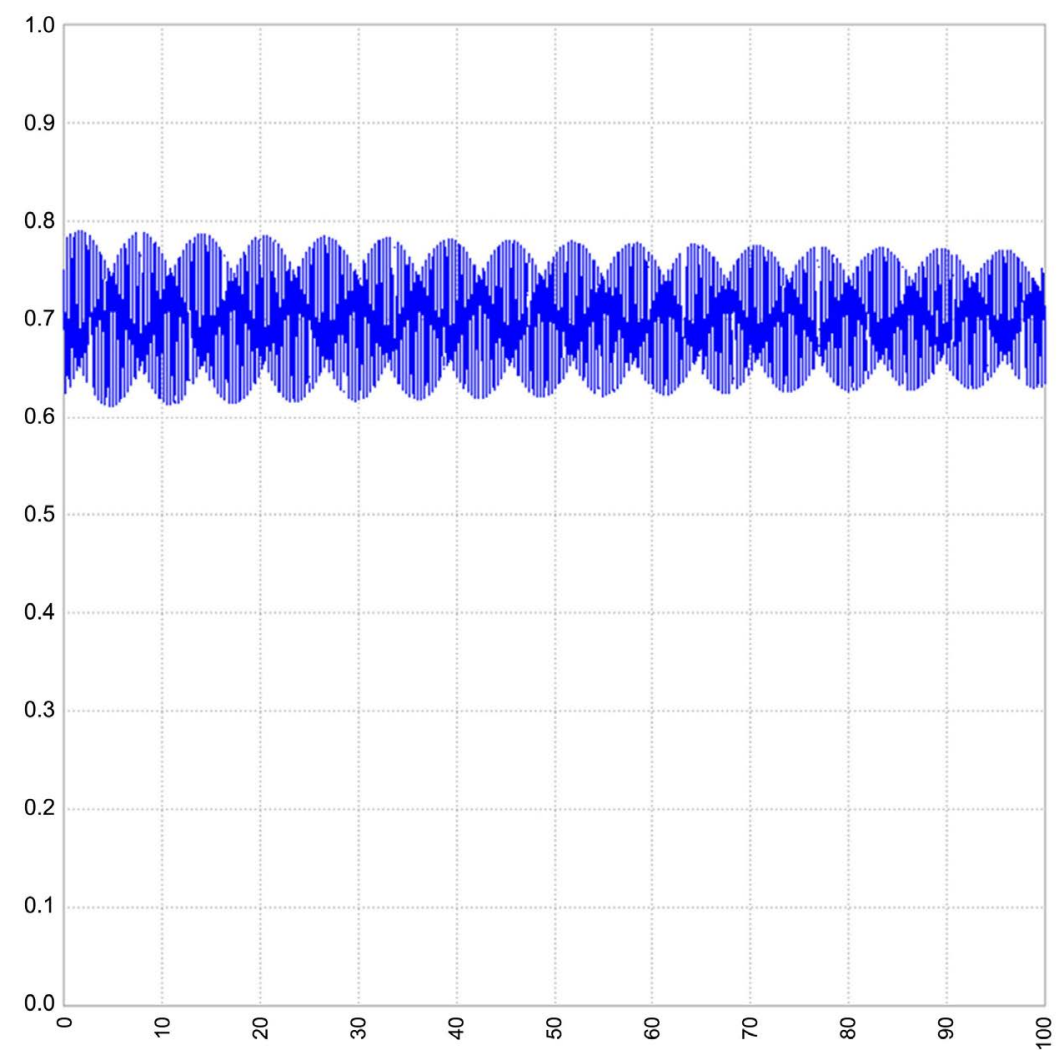

Figure 4. SSR power oscillation via 100 time cycles in p.u. 
Case a) $\omega_{S S R 1}=\omega_{\text {Nef }}$ using Equation (7), (7a) and (7b):

$$
\begin{aligned}
p(t)= & u(t) \cdot\left[i(t)+i(t)_{S S R 1}+i(t)_{S S R 2}\right]_{u, v, w} \\
= & \underbrace{u(t) \cdot i(t))_{u, v, w}}_{p_{1}(t)_{u, v, w}}+\underbrace{\left.u(t) \cdot i(t)_{S S R 1}\right)_{u, v, w}}_{p_{2}(t)_{u, v, w}}+\underbrace{\left(u(t) \cdot i(t)_{S S R 2}\right)_{u, v, w}}_{p_{3}(t)_{u, v, w}} \\
= & 3 \cdot u_{r m s} \cdot i_{r m s} \cdot \cos (\Delta \varphi) \\
& +3 \cdot u_{r m s} \cdot i_{S S R 1 r m s} \cdot \mathrm{e}^{-\alpha_{S S R 1} \cdot t} \cdot\left[\cos \left(\left(\omega_{S S R 2}\right) \cdot t-\varphi_{S S R 1}\right)\right] \\
& +3 \cdot u_{r m s} \cdot i_{S S R 2 r m s} \cdot \mathrm{e}^{-\alpha_{S S R 2} \cdot t} \cdot\left[\cos \left(\left(\omega_{N e f}\right) \cdot t-\varphi_{S S R 2}\right)\right]
\end{aligned}
$$

From Equation (10) it can be seen that the excitation of the shaft train to torsional vibrations by feeding a sub-frequency power oscillation with a torsional natural frequency $\omega_{S S R 2}$ of the shaft train. Furthermore, the sub-frequency power oscillation fed into the grid oscillates with a grid natural frequency $\omega_{\mathrm{Nef}}$.

Case b) $\omega_{S S R 1}=\omega_{S S R 2}=\omega_{S S R}$ :

$$
\begin{aligned}
p(t)= & u(t) \cdot\left[i(t)+i(t)_{S S R 1}+i(t)_{S S R 2}\right]_{u, v, w} \\
= & \underbrace{(u(t) \cdot i(t))_{u, v, w}}_{p_{1}(t)_{u, v, w}}+\underbrace{\left(u(t) \cdot i(t)_{S S R 1}\right)_{u, v, w}}_{p_{2}(t)_{u, v, w}}+\underbrace{\left(u(t) \cdot i(t)_{S S R 2}\right)_{u, v, w}}_{p_{3}(t)_{u, v, w}} \\
= & 3 \cdot u_{r m s} \cdot i_{r m s} \cdot \cos (\Delta \varphi) \\
& +3 \cdot u_{r m s} \cdot i_{S S R 1 r m s} \cdot \mathrm{e}^{-\alpha_{S S R 1} \cdot t} \cdot\left[\cos \left(\left(\omega_{N}-\omega_{S S R}\right) \cdot t-\varphi_{S S R 1}\right)\right] \\
& +3 \cdot u_{r m s} \cdot i_{S S R 2 r m s} \cdot \mathrm{e}^{-\alpha_{S S R 2} \cdot t} \cdot\left[\cos \left(\left(\omega_{N}-\omega_{S S R}\right) \cdot t-\varphi_{S S R 2}\right)\right]
\end{aligned}
$$

The two subsynchronous summands in Equation (11) add each other with the same frequency, so that in this case there is an amplification of the subsynchronous power components up to a continuous excitation.

Case c) $\omega_{S S R 1}=\omega_{N}-\omega_{N e f 1}$ :

$$
\begin{aligned}
p(t)= & u(t) \cdot\left[i(t)+i(t)_{S S R 1}+i(t)_{S S R 2}\right]_{u, v, w} \\
= & \underbrace{(u(t) \cdot i(t))_{u, v, w}}_{p_{1}(t)_{u, v, w}}+\underbrace{\left(u(t) \cdot i(t)_{S S R 1}\right)_{u, v, w}}_{p_{2}(t)_{u, v, w}}+\underbrace{\left(u(t) \cdot i(t)_{S S R 2}\right)_{u, v, w}}_{p_{3}(t)_{u, v, w}} \\
= & 3 \cdot u_{r m s} \cdot i_{r m s} \cdot \cos (\Delta \varphi) \\
& +3 \cdot u_{r m s} \cdot i_{S S R 1 r m s} \cdot \mathrm{e}^{-\alpha_{S S R 1} \cdot t} \cdot\left[\cos \left(\left(\omega_{N e f 1}\right) \cdot t-\varphi_{S S R 1}\right)\right] \\
& +3 \cdot u_{r m s} \cdot i_{S S R 2 r m s} \cdot \mathrm{e}^{-\alpha_{S S R 2} \cdot t} \cdot\left[\cos (\underbrace{\left.\left(\omega_{N}-\omega_{S S R 2}\right) \cdot t-\varphi_{S S R 2}\right)}_{\omega_{N e f 2}})\right] \\
= & 3 \cdot u_{r m s} \cdot i_{r m s} \cdot \cos (\Delta \varphi) \\
& +3 \cdot u_{r m s} \cdot i_{S S R 1 r m s} \cdot \mathrm{e}^{-\alpha_{S S R 1} \cdot t} \cdot\left[\cos \left(\left(\omega_{N e f 1}\right) \cdot t-\varphi_{S S R 1}\right)\right] \\
& +3 \cdot u_{r m s} \cdot i_{S S R 2 r m s} \cdot \mathrm{e}^{-\alpha_{S S R 2} \cdot t} \cdot\left[\cos \left(\left(\omega_{\text {Nef } 2}\right) \cdot t-\varphi_{S S R 2}\right)\right]
\end{aligned}
$$

The boundary condition introduced in Equation (12) leads to the fact that both the power oscillation fed subfrequently into the generator has a grid normal frequency $\omega_{\text {Nef1 }}$ as well as the subfrequent power oscillation fed into the grid 
with a grid natural frequency $\omega_{\mathrm{Nef} 2}$ exists.

$$
\begin{aligned}
\text { Case d) } \omega_{S S R 2} & =\omega_{N}-\omega_{N e f}: \\
p(t)= & u(t) \cdot\left[i(t)+i(t)_{S S R 1}+i(t)_{S S R 2}\right]_{u, v, w} \\
= & \underbrace{(u(t) \cdot i(t))_{u, v, w}}_{p_{1}(t)_{u, v, w}}+\underbrace{\left.u(t) \cdot i(t)_{S S R 1}\right)_{u, v, w}}_{p_{2}(t)_{u, v, w}}+\underbrace{\left(u(t) \cdot i(t)_{S S R 2}\right)_{u, v, w}}_{p_{3}(t)_{u, v, w}} \\
= & 3 \cdot u_{r m s} \cdot i_{r m s} \cdot \cos (\Delta \varphi) \\
& +3 \cdot u_{r m s} \cdot i_{S S R 1 r m s} \cdot \mathrm{e}^{-\alpha_{S S R 1} \cdot t} \cdot\left[\cos \left(\left(\omega_{N}-\omega_{S S R 1}\right) \cdot t-\varphi_{S S R 1}\right)\right] \\
& +3 \cdot u_{r m s} \cdot i_{S S R 2 r m s} \cdot \mathrm{e}^{-\alpha_{S S R 2} \cdot t} \cdot\left[\cos \left(\left(\omega_{N e f}\right) \cdot t-\varphi_{S S R 2}\right)\right]
\end{aligned}
$$

Equation (13) shows that the sub-synchronous power oscillation fed into the grid under this assumption occurs with a grid natural frequency.

\subsection{Evaluation of the Results}

The feed in of sub-frequency currents from the grid into the generator of a conventional turboset can excite the shaft train involved to torsional oscillations, see [3]. This depends largely on the prevailing frequency conditions. The excited torsional oscillations induce synchronous generated voltages in the generator stator which in turn lead to sub-frequency currents. Each of the generated sub-frequency currents causes in turn subsynchronous power oscillations which are superimposed on each other. Whether these power oscillations remain unchanged in their effects as a function of time or decay with time depends on the damping factor.

\section{Conclusion}

Since several decades it is well-known that grid faults are able to excite torsional oscillations of turbo set shaft trains, see [4] [5] [6] [7]. By the implementation of an increasing number of converter systems to the grid, the exciation mechanism will change and become more complex. According to this, deeper investigations in all directions are necessary, e.g. measurements of torsional oscillations during operation ([8] [9]), but also theoretical simulations of the system "Grid-Converter-electrical Generator", see [3]. In general, the interaction between the grid frequency, the natural electrical grid frequencies and the mechanical torsional natural frequencies has to be considered in a more effective way. One important question in general will be, if the well-known protection systems ([10]) for turbo sets are able to deal with the new grid situation without any restrictions.

\section{Conflicts of Interest}

The author declares no conflicts of interest regarding the publication of this paper.

\section{References}

[1] Piwko, R.J. and Larsen, E.V. (1982) HVDC System Control for Damping of Sub- 
synchronous Oscillations, Palo Alto, California. 2352-2360.

[2] Working Group 14.05 (1997) Interaction between HVDC Convertors and Nearby Synchronous Machines. Cigre.

[3] Exnowski, S. andHumer, M. (2018) Subsynchronous Resonances in the Shaft Train of Large Turbosets and High Voltage DC Transmission. 23th International Conference on Electrical Machines, Alexandroupoli, Greece, 3-6 September 2018, 414-418. https://doi.org/10.1109/ICELMACH.2018.8507243

[4] Humer, M., Salzig, C. and Wirsen, A. (2020) Monitoring von Torsionsschwingungen als Folge von Netzinteraktionen, Essener Turbogeneratorentagung "Generatoren in konventionellen Kraftwerken, Windparks und Wasserkraftwerken”, Haus der Technik Essen, 18-19 February 2020, Germany.

[5] Humer, M. (2012) Entstehung und Messung von Torsionsschwingungen an Kraftwerksturbosätzen. AKIDA 2012, Aachen, Germany, 14-15 November 2012, 327-338.

[6] Humer, M. and Golebiowski L. (2012) Subsynchrone Resonanzen im System Turbosatz-Netz/Entstehung und Auswirkung auf den Turbosatz, Haus der Technik, Seminar "Turbogeneratoren im Kraftwerk", Essen, Germany.

[7] Kundur, P. (2017) Power System Stabilty and Control. McGraw-Hill, New York.

[8] Wirsen, A., Lang, P. and Humer, M. (2004) Systems for Monitoring and Analysing Torsional Vibrations in Turbine Generator Shaft Lines. Conference Proceedings of the 16th International Conference on Electrical Machines, Krakow, Poland, 5-8 September 2004, 855-856.

[9] Humer, M. and Kulig, S. (2003) Measurement and Assessment of Torsion Oscillations in Turbogenerators by Using a Torque Sensor and Robust Observer. IEEE Industrial Electronics Society, Roanoke, Virginia, USA.

[10] Humer, M., Golebiowski, L. and Kulig, S. (2011) Reduzierung der Turbosatzbeanspruchungen durch den Einbau des Kraftwerksentkupplungsschutzes. VGB PowerTech 8/2011, 91, 54-59. 